ZUR A. GENEBIS.

vorschwebte, war natürlich die grösse, der langgestreckte anblick des herschaftlichen gastseli.

Mir scheint, dass diese deutung eine lücke in Braunes erörterungen ausfüllt, die notwendig der ausfüllung bedurfte. Gastseli als 'haus für gäste', daher 'herrenhaus' ist doch an sich viel weniger einleuchtend als 'domus comitum'. Die gelegentlichen gastereien sind für den altgerm. fürstenhof weit weniger bezeichnend als das alltägliche schmausen und trinken der streitbaren hausgenossenschaft. Der kleine bauer dagegen sass einsam mit seiner familie zu hause. Als illustration fällt einem sogleich Heorot ein, Hrólfr kraki, Jormunrekr in den Hamðismál, Fródi's tod in der Hrólfssaga, um weiterer nordischer beispiele zu geschweigen.

BRESLAU, 3. märz 1907.

G. NECKEL.

\title{
ZUR ALTSÄCHSISCHEN GENESIS.
}

V. $287 \mathrm{f}$. ist überliefert: âhtfugal sang fora daga huoam.

Die zahlreichen besserungsvorschläge sind in Behaghels ausgabe s. 238 verzeichnet. In der überzeugung, dass keiner völlig befriedigt, wage ich im engsten anschluss an die hs. einen neuen: man lese hruomag 'fröhlich' statt des sinnlosen huoam!') Das wort steht im Heliand v.944f. than eu lango scal| uuesan euua hugi hrômag und v.4926 thiu hêri uucurठ thes sô hrômeg (in Behaghels ausgabe); es ist ferner im ahd. als houomac, -îg und im ae. als hrêmig überliefert. Das fröhliche krähen des hahnes gerade vor dem schrecklichen untergange der stadt ist ein für den dichter der Genesis charakteristischer zug! - Dass in v. 287 eine umstellung vorzunehmen sei, davon bin ich nach wie vor überzeugt.

v. 321 ff. lauten in der hs.:

Sôdomarîki: that is ênig theg ni ginas,

al uuaro farspildit

ac sô bidoơ it an dôtsêu, sô it noh te daga stendit, fluodas gifullit.

1) Auch Martin, QF. 100, 48 schlägt jetzt hruomag vor (Corr.-note). 
Mit Cosijn seg für theg einsetzend möchte ich jetzt nur noch bidod in bidrôs 'fiel, sank' bessern, um einen tadellosen vers und sinn zu erhalten. Dann werden alle von Bèhaghel a.a.o. s. 239 f. angeführten änderungen überflüssig! Bidrôs steht wie bivallan Gen. 233 und das häufige bifällan im Heliand und ahd., befĕallan im ae. = fallan, desgl. bisank Gen. 320 und bisunki Hel. 5692 für einfaches sinkanz; es entspricht genau dem ae. bedreosan, das allerdings nur im part. prt. bedroren 'gefallen, beraubt' erscheint. doð für dros ist offenbar durch das folgende doð veranlasst! Denkbar wäre auch bidôf; -dôt $=$ ae. bedeaf 'tauchte'.

\section{STAIMBORT CHLUDUN.}

Wenn ich diese alte crux des ahd. Hildebrandsliedes (v. $65 \mathrm{~b}$ nach Braunes Ahd. leseb.5 s. 79) hier von neuem behandle, so geschieht dies in der hoffnung, eine noch nicht vorgebrachte besserung des ersten wortes rechtfertigen zu können. Man hat staim bald als stein 'edelstein' oder 'farbe' (so Meissner in der Zs. fda. 47, $400 \mathrm{ff}$.), bald als steim 'gewühl, gedrä̀nge' (vgl. Wadstein, Göteborgs högskolas årskrift 1903) gefasst: beiden erklärungen widerspricht schon die sonstige bezeichnung des alten diphthongen ai, der teils als $e i$, teils als $e, e, \infty$ oder ae, aber niemals als ai erscheint! Da der text des gedichtes auch sonst durch schreibfehler entstellt ist, möchte ich hier ebenfalls einen solchen annehmen und das in der hs. nicht ganz deutliche staim als verlesung oder verschreibung für sturm 'kampf' ansehen. Sturmbort 'kampfschild' würde vorzüglich passen (vgl. Wadstein a.a.o. s. $38 \mathrm{f}$.) und sich den zahlreichen mhd. compositis mit sturm- anschliessen; chludun ist gewiss von Wackernagel richtig in chlubun 'kloben, spalteten' (trans.) gebessert und wenn wir noch mit. Klaeber, Mod. Lang. Notes 21, no. 4, s. 110 in v. 63 b ascki für asckim lesen, lautet die ganze stelle (v. $63 \mathrm{ff}$.) mit berichtigter zeichensetzung:

dô lệttun sê pêrist ascki scrîtan scarpên scârim: dat in dêm sciltim stônt!

65 dô stôpun tôsamane, sturmbort chlubun, heuwun harmlîcco huîttę scilti. 